\title{
LES MODĖLES STATISTIQUES DE DESCRIPTION DE L'HABITAT HYDRAULIQUE : DES OUTILS POUR L'ÉCOLOGIE.
}

\author{
N. LAMOUROUX $(1,2)$
}

(1) URA CNRS 1974, "Ecologie des Eaux Douces et des Grands Fleuves", Université Lyon 1, 69622 Villeurbanne Cedex, France.

(2) Compagnie Nationale du Rhône, 2 rue André Bonin, 69316 Lyon Cedex 04, France.

\begin{abstract}
RÉSUMÉ
Quantifier les impacts écologiques des aménagements de rivière et de leur gestion nécessite une description fine de l'habitat hydraulique. Les modèles statistiques de description de l'habitat hydraulique en cours de développement ont pour objet d'estimer la distribution des habitats locaux dans un segment de rivière à partir de ses caractéristiques moyennes. Ils font ainsi le lien entre l'échelle de travail des hydrauliciens fluviaux (échelle du segment), et les besoins des écologues (échelle locale). Leur simplicité d'utilisation ouvre de larges perspectives d'application, en particulier pour la gestion des débits réservés. Leur généralité contribue à mieux cerner le rôle de l'habitat hydraulique dans la complexité de l'écosystème fluvial.
\end{abstract}

\section{STATISTICAL MODELS OF THE HYDRAULIC HABITAT : TOOLS FOR ECOLOGY.}

\begin{abstract}
Quantitative estimates of the ecological impacts of river management require a description of the hydraulic local habitat. Statistical hydraulic models aim at providing estimates of the frequency distribution of local hydraulic variables in a stream reach, as a function of average characteristics of the reach. Such models bridge the gap between the working scale of fluvial hydrautics (the reach scale), and the needs of ecologists (local scale). Because they use simple input variables, they offer large possibilities of application, in particular for instream flow assessments. Statistical models reflect general trends common to complex fluvial systems and therefore contribute to explain the role played by the physical characteristics of the habitat.
\end{abstract}

\section{INTRODUCTION}

Ne serait-ce qu'en France, plus de 400 barrages et autres aménagements hydrauliques transforment l'hydrologie ou la morphologie des cours d'eau. Ces impacts concernent un ensemble de domaines variés : transformation de la dynamique fluviale, des variables hydrauliques locales, des connexions entre le cours d'eau et ses annexes, des phénomènes de transport et d'érosion, du niveau des nappes, du paysage, et bien d'autres encore. L'un et/ou l'autre de ces aspects peut être limitant pour le système vivant. II convient donc de construire des outils simples et généraux de quantification de ces impacts, afin de les prendre en compte sur un nombre maximal de sites, et d'adopter des solutions plus raisonnées.

En particulier, le rôle important joué par les variables hydrauliques locales sur les populations de poissons et d'invertébrés a récemment été démontré (BOVEE, 1978 ; STATZNER et al., 1988 ; SOUCHON et al., 1989). Dans ce cadre, les besoins en matière de description des variables hydrauliques comportent deux exigences particulières :

(1) Dans un premier temps, les modèles doivent permettre une estimation fiable des variables hydrauliques à l'échelle locale (échelle des organismes ou des prélèvements) à laquelle les organismes montrent des préférences. 
(2) Dans un second temps, ils doivent être simplement utilisables, afin de pouvoir tester le plus largement possible les liens entre l'habitat hydraulique et les caractéristiques des peuplements. En effet, la complexité des systèmes d'eaux courantes impose de confronter les données relevées ou modélisées sur un nombre maximal de sites, afin de cerner les phénomènes généraux communs à l'ensemble de ces sites.

(1) La première exigence se heurte à un certain nombre de barrières entre les travaux des écologues et les travaux des hydrauliciens. Tout d'abord, si les besoins des écologues relèvent de l'échelle locale, l'hydraulique fluviale a cherché à résoudre des problèmes qui relèvent d'une échelle plus globale : dimensionnement de canaux, estimation de lignes d'eau et de débits, propagation des crues. Les modèles déterministes largement développés par les hydrauliciens pour ces besoins résolvent les équations de la physique, mais diminuent en précision d'autant plus que l'on s'éloigne d'une géométrie simple et d'un écoulement uniforme. L'utilisation de tels modèles en rivière permet une estimation correcte des vitesses et hauteurs moyennes pour les écoulements calmes. Par contre, l'estimation des variables ponctuelles qui intéressent les écologues est irréaliste (OSBORNE et al., 1988). Enfin, de même que la prise de conscience de l'importance des contraintes hydrauliques a été tardive en écologie, le cloisonnement des disciplines n'a pas poussé les hydrauliciens à développer des modèles spécifiques aux besoins des écologues.

(2) La seconde exigence se heurte à la complexité de la mise en oeuvre des modèles hydrauliques. En effet, par-delà les problèmes de précision ci-dessus évoqués, les modèles hydrauliques déterministes nécessitent, pour leur application en rivière, un relevé topographique précis du lit, de nombreuses mesures hydrologiques, et un calage du modèle par l'expert. Cette lourdeur rend coûteuse la reconstitution ou la prévision de chroniques d'habitat hydraulique.

La modélisation statistique de l'habitat dans un segment de rivière cherche à répondre à ces deux exigences. Les objectifs de cette note de synthèse sont (1) de présenter les premiers développements de modèles statistiques qui visent à estimer les variables hydrauliques locales, (2) de démontrer, à l'aide de simulations sur différents cours d'eau, la simplicité d'utilisation de ces modèles, et (3) d'évoquer les perspectives offertes par l'approche statistique pour typer les rivières et évaluer a priori leur sensibilité aux modifications de débit.

\section{LA MODÉLISATION STATISTIQUE DE L'HABITAT : UNE ESTIMATION SYNTHÉTIQUE DES HABITATS LOCAUX DANS UN SEGMENT DE RIVIERRE}

Les modèles statistiques visent à estimer la distribution en fréquence des variables ponctuelles dans un segment de rivière, en fonction des caractéristiques moyennes de ce segment. Le segment est ici défini comme quelques successions de type radier-mouille ("reach", dans NEWBURY et GABOURY (1993)). A l'inverse des modèles hydrauliques déterministes, les modèles statistiques ne cherchent pas à cartographier les variables hydrauliques à l'intérieur du segment, mais à prédire leur distribution statistique dans le segment indépendamment de leur répartition spatiale. Ils visent ainsi à quantifier et fiabiliser l'intuition de l'expert qui, à partir d'une visualisation des caractéristiques moyennes de la rivière, peut estimer la fréquence des zones rapides ou des zones plus calmes.

Ces modèles permettent actuellement d'estimer la distribution statistique des contraintes au fond (LAMOUROUX et al., 1992) et des vitesses ponctuelles (LAMOUROUX et al., 1995) dans un segment de rivière à morphologie peu transformée mais dont le débit peut être fortement modifié. La contrainte au fond est une force qui contrôle l'habitat benthique, tandis que la vitesse ponctuelle concerne essentiellement l'habitat piscicole. Les modèles statistiques prédisent ces variables locales en fonction de variables moyennes sur le segment, en particulier le nombre de Froude :

$$
\mathrm{Fr}=\mathrm{Q} / \mathrm{g}^{0.5 \mathrm{H}^{1.5} \mathrm{~L}}
$$

où $Q$ est le débit, g la gravité, $H$ la hauteur d'eau moyenne, et $L$ la largeur mouillée moyenne. Le nombre de Froude quantifie l'importance des phénomènes d'inertie par rapport aux phénomènes gravitaires. L'influence sur les distributions de vitesse de la granulométrie relative $\mathrm{D} / \mathrm{H}$, où $\mathrm{D}$ est la taille moyenne des éléments du lit, a également été 
mise en évidence. Cette variable n'était pas disponible sur les cours d'eau concernés par les mesures de contraintes, mais sa prise en comple devrait permettre d'élargir le champ d'application du modèle.

Si la prédiction statistique des contraintes et des vitesses est possible, c'est parce que leurs distributions ont des formes caractéristiques (Figures 1 et 2). Ces distributions sont le plus souvent bimodales, traduisant l'alternance de groupes de faibles vitesses ou faibles contraintes, avec des groupes de vitesses ou contraintes plus fortes. Cette bimodalité a pour origine une variabilité transversale et longitudinale des conditions hydrauliques dans le segment, ainsi qu'une variabilité plus diffuse liée au rôle des obstacles (LAMOUROUX et al., 1995). Pour de fortes valeurs du Froude, la bimodalité s'estompe et les distributions de vitesses et contraintes tendent vers des lois de Gauss. Par contre, de fortes valeurs de granulométrie relative renforcent la bimodalité.

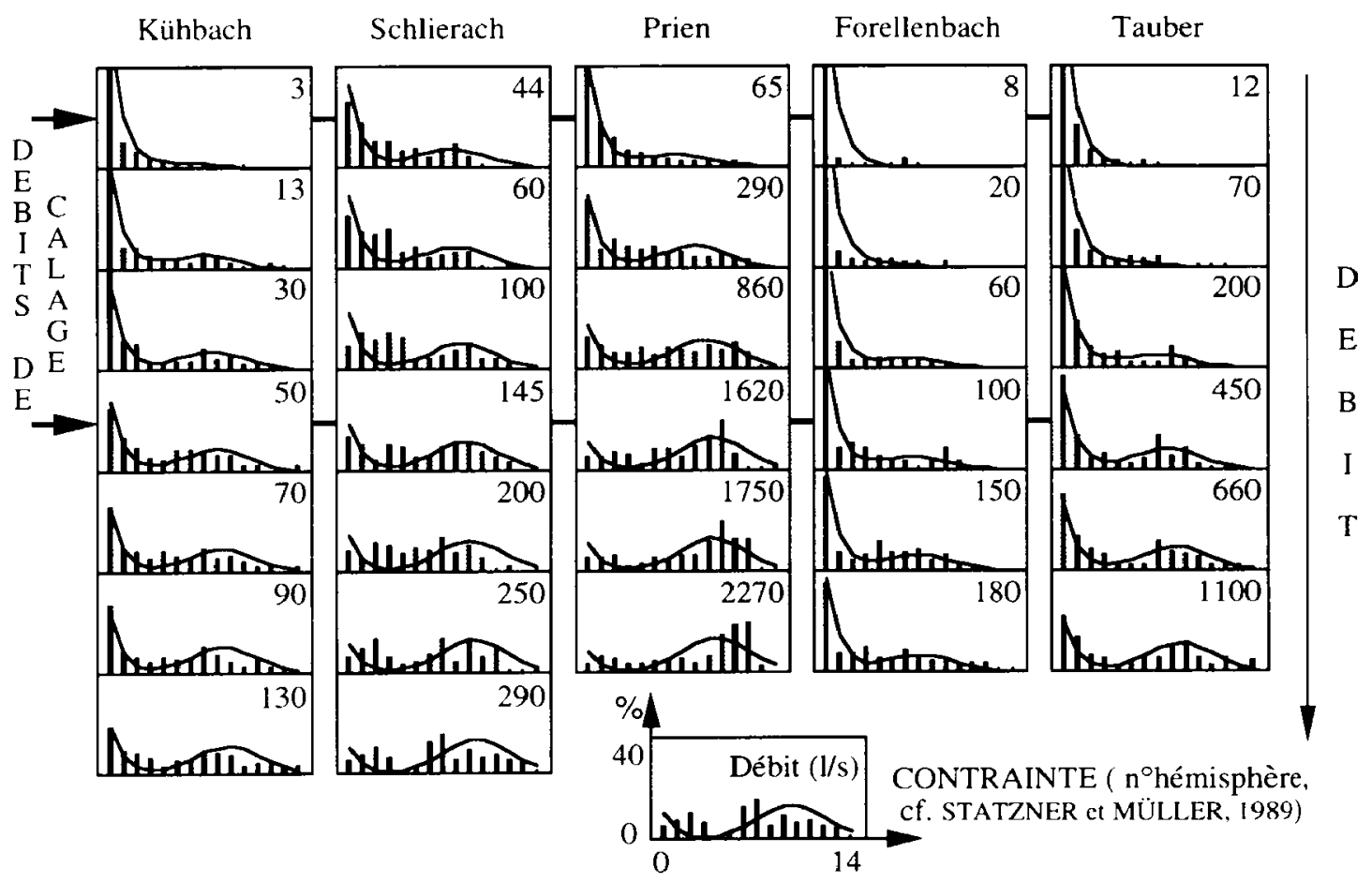

Figure 1 : Distributions en fréquence de la contrainte au fond sur 5 segments de rivières, observées (bâtons) et modélisées à l'aide d'un modèle statistique (lignes). Des mesures simples (cf. texte) ont été effectuées aux deux débits de calage indiqués par des flèches (cf. LAMOUROUX et al. (1992) pour une description plus détaillée des 5 segments de rivières).

Figure 1 : Measured (bars) and predicted (lines) frequency distributions of local shear stress in 5 different stream reaches. One hundred local depths and 15 local widths (see text) were measured at 2 different discharges indicated by an arrow (see LAMOUROUX et al. (1992) for further information concerning the 5 reaches).

\section{CONVIVIALITÉ DES MODĖLES STATISTIQUES}

Les modèles statistiques estiment les variables locales en fonction de caractéristiques moyennes du segment : nous avons mentionné le rôle de $Q, H$, $L$ et $D$. Les variables d'entrée sont donc des variables simples.

Dans le cas de la recherche d'impacts de changement hydrologiques, et notamment pour la recherche de débits réservés optimaux à l'aval d'ouvrages, il est nécessaire de prévoir au préalable l'évolution temporelle de ces variables moyennes avec le débit. Ceci 


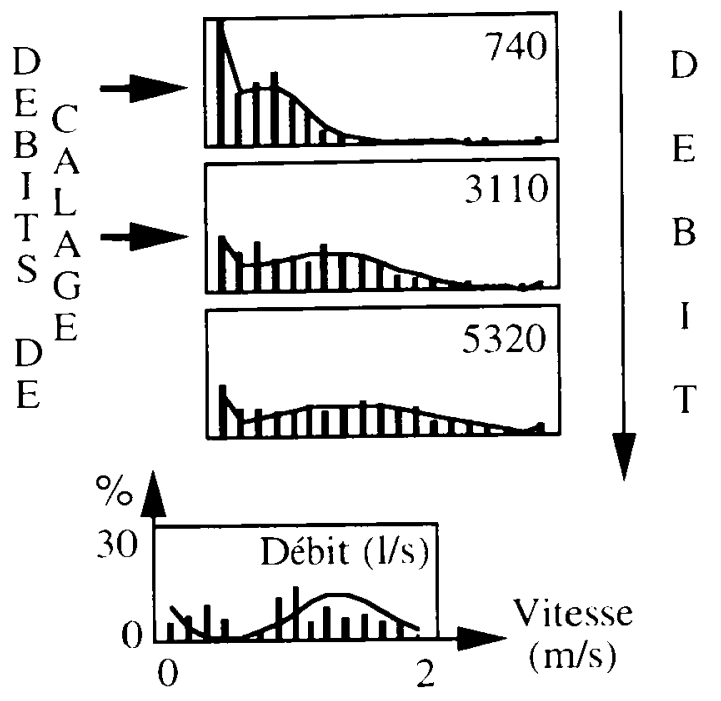

Figure 2 : Distributions en fréquence de la vitesse ponctuelle dans un segment de l'Arc, observées (bâtons) et modélisées à l'aide d'un modèle statistique (lignes). Des mesures simples (cf. texte) ont été effectuées aux deux débits de calage indiqués par des flèches (cf. LAMOUROUX et al. (1995) pour une description plus détaillée de l'Arc).

Figure 2 : Measured (bars) and predicted (lines) frequency distributions of point velocity in a reach of the Arc river. One hundred local depths and 15 local widths were measured at 2 different discharges indicated by an arrow (see text). In addition, the dominant roughness was noted in 15 places (see LAMOUROUX et al. (1995) for further information concerning the reach).

est possible à l'aide de modèles déterministes peu précis lorsque la pente de l'écoulement n'est pas trop élevée. Cependant, les évolutions de $H$ et $L$ en fonction de $Q$ peuvent être obtenues plus simplement encore à l'aide de formules empiriques ou de mesures existantes. Par exemple, de simples mesures à deux débits différents permettent de caler des lois de type puissance, dont la généralité a largement été démontrée (LEOPOLD et al., 1964), et qui pour un segment s'expriment sous la forme :

$$
\mathrm{H}=\mathrm{a} \mathrm{Q}^{\mathrm{b}} \quad \mathrm{L}=\mathrm{c} \mathrm{Q}^{\mathrm{d}}
$$

où $\mathrm{a}, \mathrm{b}, \mathrm{c}, \mathrm{d}$ sont des constantes qui dépendent du segment. De telles estimations des caractéristiques moyennes du segment puis l'utilisation d'un modèle statistique permettent d'estimer les variables hydrauliques locales à différents débits sans relevé topographique précis, sans calage de modèles hydrauliques, et sans mesures de vitesses ou de contraintes.

La figure 1 est un exemple de simulation de l'évolution de la distribution des contraintes au fond avec le débit. L'expérience a été répétée sur 5 cours d'eau bavarois provenant de bassins versants différents, pour lesquels les distributions de contraintes ont été observées à 6 ou 7 débits différents (LAMOUROUX et al., 1992). Sur chaque cours d'eau, de simples mesures ont été réalisées à deux débits différents : 100 hauteurs d'eau et 15 largeurs mesurées au hasard sur le segment. Ces mesures ont permis un très rapide calage de relations $H(Q)$ et $L(Q)$ (Equation 2). On constate visuellement sur la figure 1 que le modèle statistique de description des contraintes (LAMOUROUX et al., 1992) suffit alors à estimer de manière satisfaisante l'évolution de la distribution des contraintes au fond avec le débit. Les prévisions restent très proches des observations pour des débits de l'ordre du double du débit maximal échantillonné.

Une démarche identique permet de prédire avec précision la distribution des vitesses ponctuelles sur une rivière alpine, l'Arc, à trois débits différents (Figure 2). Pour cette simulation, nous avons également estimé la granulométrie moyenne du segment à partir d'observations du substrat dominant en 15 points choisis au hasard. 


\section{PERSPECTIVES}

Les modèles statistiques de description de l'habitat local ont pour objet d'être couplés à des modèles de préférence des organismes pour ces habitats locaux, afin de prévoir les impacts de la gestion du cours d'eau sur le système vivant. Les modèles de préférence d'invertébrés pour la contrainte au fond sont d'ors et déjà utilisables avec les modèles statistiques qui la prédisent (STATZNER et BORCHARDT, 1994). En ce qui concerne les poissons, POUILLY (1994) a montré l'intérêt de modèles de prédiction du peuplement en fonction de distributions de vitesses, hauteurs et substrats ponctuels dans la zone de prélèvement. Ces modèles sont linéaires en fonction des effectifs de classes des trois variables physiques (à ne pas confondre avec une linéarité en fonction des variables elles-mêmes). Ils sont bâtis à l'échelle des prélèvements, mais leur linéarité autorise à les utiliser à l'échelle du segment entier. Ils seront donc utilisables avec des modèles statistiques univariés qui prédisent les distributions de vitesse et de hauteur d'eau dans le segment. Par contre, l'utilisation de modèles de préférence piscicoles non linéaires imposera, elle, l'utilisation de modèles statistiques multivariés qui prédisent la distribution dans le segment du triplet (vitesse, hauteur, substrat).

Outre les perspectives de couplage avec les modèles de préférence développés à l'échelle locale, l'approche statistique propose de nouvelles perspectives de description de I'habitat à l'échelle du segment de rivière. En effet, à la différence des modèles hydrauliques déterministes, les modèles statistiques ici présentées indiquent le lien entre les caractéristiques moyennes du segment et l'habitat local. II en résulte qu'ils contribuent non seulement à décrire l'habitat local, mais également à cerner le rôle des caractéristiques moyennes du segment sur les conditions locales. Les modèles statistiques constituent donc une passerelle entre deux échelles de travail complémentaires : l'échelle locale à laquelle les préférences d'habitat des organismes sont observées, et l'échelle du segment à laquelle le gestionnaire agit mais où le rôle de l'habitat hydraulique s'isole difficilement du fait de la complexité du système (figure 3 ).

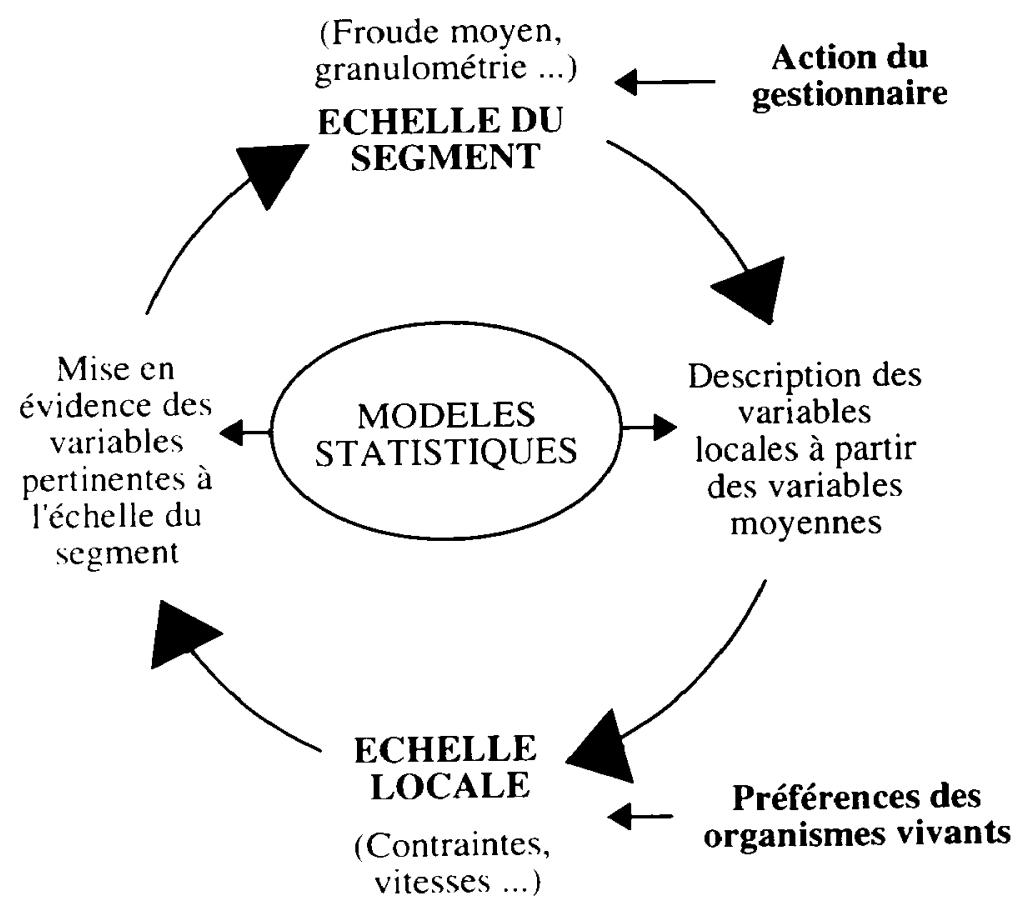

Figure 3 : Les modèles statistiques de description de l'habitat hydraulique : une passerelle entre l'échelle du segment et l'échelle locale.

Figure 3 : Statistical modelling of the hydraulic habitat : bridging the gap between the reach scale and the local scale. 
A l'échelle locale comme à l'échelle du segment, la simplicité des variables d'entrée des approches statistiques peut faciliter les études d'impact de la gestion ainsi que la reconstitution de chroniques d'habitat dans un cours d'eau donné en fonction de l'espace ou du temps (STATZNER et BORCHARDT, 1994). De telles reconstitutions sur différents sites qui ont fait l'objet de suivis biologiques à long terme (cf. par exemple SANLAVILLEBOISSON et al., 1995) aideront à rechercher les périodes structurantes et les conditions d'habitat limitantes pour le système vivant. Elles contribueront à quantifier le rôle de l'hydraulique sur les peuplements, et à établir une typologie des différents segments de rivière qui tient compte de l'habitat.

\section{CONCLUSION}

Les modèles statistiques de description de l'habitat sont des outils en cours de développement qui ont pour objet de prédire la valeur des variables hydrauliques locales, mais contribuent également à la recherche de descripteurs pertinents de l'habitat à l'échelle du segment. Deux exemples ont montré qu'ils permettent d'estimer l'évolution de variables hydrauliques avec le débit à partir de mesures peu coûteuses. Cette simplicité d'utilisation laisse envisager de larges possibilités d'application pratique, d'exploitation de données existantes, et de recherche de phénomènes communs à différents sites expérimentaux.

Les limites actuelles des modèles statistiques sont multiples. Tout d'abord, leur développement se borne actuellement à la prédiction de contraintes au fond et de vitesses, sur des cours d'eau de débits inférieurs à environ $20 \mathrm{~m}^{3} / \mathrm{s}$. Ensuite, il va de soi que ces modèles généraux sont limités en précision par la présence de singularités hydrauliques fortes sur un segment (débris, calibrage...), puisqu'ils s'intéressent à mettre en évidence les points communs entre différents sites. Enfin, si les modèles statistiques univariés peuvent ou pourront être couplés avec certains modèles de préférence d'invertébrés et de poissons, le développement de modèles multivariés ouvrira largement les perspectives de couplage.

Malgré ces limites, il faut rappeler que la complexité du fonctionnement d'un cours d'eau rend impossible une étude spécifique et poussée sur chaque site. C'est pourquoi la quantification de tendances générales communes à un ensemble de cours d'eau est une voie qui mène vers des outils simples et objectifs, qui permettent de peser au mieux les divers impacts d'un aménagement et de sa gestion. Une telle approche permet d'unifier une multitude de relations a priori différentes entre hydraulique et écologie, qui sont mises en évidence sur différents sites mais ne sont pas transposables, ce qui laisse le gestionnaire dans l'embarras (BEECHER, 1990).

\section{REMERCIEMENTS}

Je remercie Yves SOUCHON, Michel LARINIER et un correcteur anonyme d'avoir soulevé de manière constructive des points de discussion importants. Eric HÉROUIN et Bernard STATZNER ont critiqué avec pertinence une première version de cette note. Les graphiques ont été réalisés à l'aide du logiciel ADE (DOLEDEC et CHESSEL, 1993). Les travaux ont été financés par la Compagnie Nationale du Rhône, le Ministère de la Recherche, et le Programme Interdisciplinaire de Recherche 'Poisson' et 'Hydrosystème fluvial'.

\section{BIBLIOGRAPHIE}

BEECHER H.A., 1990. Standards for instream flow. Rivers, 1 (2), 97-109.

BOVEE K.D., 1978. Probability of use criteria for the family salmonidae. Tech. Rep. FWS/OBS-78/07. U.S. Fish and Wildlife Serv., Fort Collins, Colorado, 90 p.

DOLEDEC S., CHESSEL D., 1993. Programmathèque ADE. Analyses multivariées et expression graphique des données environnementales. Version 3.6. Université Lyon 1, Villeurbanne, France, $750 \mathrm{p}$. 
LAMOUROUX N., STATZNER B., FUCHS U., KOHMANN F., SCHMEDTJE U., 1992. An unconventional approach to modelling spatial and temporal variability of local shear stress in stream segments. Water Resources Research, 28, 3251-3258.

LAMOUROUX N., SOUCHON Y., HEROUIN E., 1995. Predicting velocity distributions in stream reaches. Water Resources Research, 31, 2367-2375.

LEOPOLD L.B., WOLMAN M.G., MILLER J.P., 1964. Fluvial processes in geomorphology. W.H. Freeman \& Co., San Francisco, California, 522 p.

NEWBURY R., GABOURY M., 1993. Exploration and rehabilitation of hydraulic habitats in streams using principles of fluvial behavior. Freshwater Biology, 29, 195-210.

OSBORNE L.L., WILEY M.J., LARIMORE R.W., 1988. Assessment of the water surface profile model : Accuracy of predicted instream fish habitat conditions in low-gradient, warmwater streams. Regulated Rivers : Research and Management, 2, 619-631.

POUILLY M., 1994. Relations entre l'habitat physique et les poissons des zones à cyprinidés rhéophiles dans trois cours d'eau du bassin rhodanien : vers une simulation de la capacité d'accueil pour les peuplements. Thèse de Doctorat, Université Lyon 1, Cemagref BEA-LHQ, 256 p.

SANLAVILLE-BOISSON C., OLIVIER J.M., SIMIER M., CHANGEUX T., POIZAT G., TORRE F., BELLIARD J., 1995. Les bases de données : outils de stockage et d'exploitation des échantillonnages piscicoles. Bull. Fr. Pêche Piscic., 337/338/339.

SOUCHON Y., TROCHERIE F., FRAGNOUD E., LACOMBE C., 1989. Les modèles numériques des micro-habitats des poissons : application et nouveaux développements. Revue des sciences de l'eau, 2, 807-830.

STATZNER B., GORE J.A., RESH V.H., 1988. Hydraulic stream ecology : observed patterns and potential applications. Journal of the North American Benthological Society, 7 (4), 307-360.

STATZNER B., MÜLLER R., 1989. Standard hemispheres as indicators of flow characteristics in lotic benthos research. Freshwater Biology, 21, 445-459.

STATZNER B., BORCHARDT D., 1994. Longitudinal patterns and processes along streams : Modelling ecological responses to physical gradients. In GILLER P.S., HILDREW A.G., RAFFAELLI D.G. (eds), Aquatic ecology: scale, pattern and process, 113-140, Blackwell Scientific Publications, Oxford. 